\title{
Downward jetting of a dynamic Leidenfrost drop
}

\author{
Sang-Hyeon Lee $\odot,{ }^{1}$ Maaike Rump $\odot,{ }^{2}$ Kirsten Harth $\odot,{ }^{2,3}$ Minwoo Kim $\odot,{ }^{1}$ Detlef Lohse $\odot,{ }^{2,4}$ \\ Kamel Fezzaa $\odot,^{5}$ and Jung Ho Je $\odot^{1, *}$ \\ ${ }^{1} X$-ray Imaging Center, Department of Materials Science and Engineering, Pohang University of Science \\ and Technology, Cheongam-Ro, Nam-Gu, Pohang 37673, Republic of Korea \\ ${ }^{2}$ Physics of Fluids, University of Twente, 7500NB Enschede, The Netherlands \\ ${ }^{3}$ Institute of Physics, Otto von Guericke University Magdeburg, 39106 Magdeburg, Germany \\ ${ }^{4}$ Max Planck Institute for Dynamics and Self-Organization, Göttingen, Germany \\ ${ }^{5}$ X-ray Science Division, Advanced Photon Source, Argonne National Laboratory, \\ 9700 South Cass Avenue, Argonne, Illinois 60439, USA
}

(Received 13 January 2020; accepted 16 June 2020; published 17 July 2020)

\begin{abstract}
Jetting is a universal phenomenon frequently observed in nature and industries, for instance, in rain drop impact, inkjet printing, spray cooling, fuel atomization, etc. In drop impact on a superheated surface, we observe the formation of a vapor cavity beneath the dynamic Leidenfrost drop and a consecutive downward ejection of a jet into the cavity using ultrafast $x$-ray phase contrast imaging. We reveal that the cavity is induced mostly by the retraction of the drop and the jetting is caused by the convergence of capillary waves along the liquid-cavity interface. We find a jetting criterion based on the viscous damping of capillary waves: $\left[\mathrm{OhWe}^{2}\right] \leqslant 66 \pm 10$. These results can provide important insight that leads to understanding and modeling of jets in nature.
\end{abstract}

DOI: 10.1103/PhysRevFluids.5.074802

\section{INTRODUCTION}

Jetting, the formation of a liquid jet, is a universal phenomenon frequently observed in nature and industries such as in rain drop impact [1], inkjet printing [2], spray cooling [3], and fuel injection in engines $[4,5]$. Jetting has been thus studied more than one century since the pioneering work of Worthington [6-8].

Much of the research on jetting has been performed for convenience on drop impact on a solid or liquid substrate at room temperature [9-15]. Here jetting occurs only upward from the liquid drop into the air due to convergence of capillary waves generated on the liquid-air interface by drop impact [16-18]. At this point, the thickness of the gas layer, the air film trapped between the liquid drop and the substrate, is very thin $(<\mu \mathrm{m})$ at room temperature [19,20]. In contrast, the thickness of the gas layer, the vapor film evaporated from the liquid drop, can be large, up to $>10 \mu \mathrm{m}$ at a sufficiently superheated surface [21-25], possibly levitating the liquid drop, which is the so-called Leidenfrost phenomenon. The large thickness of the vapor film brings up, together with capillary waves generated on the liquid (L)-vapor (V) interface [21], an interesting question in the Leidenfrost phenomenon: Can jetting occur downward from the L-V interface underneath the Leidenfrost drop?

One good way to answer the question above is to directly visualize the L-V interface profiles with sufficiently high spatial and temporal resolution, which is, however, a challenge in conventional optical imaging due to strong light reflection and scattering of visible light $[20,25,26]$. Interferometry

\footnotetext{
*jhje@postech.ac.kr
} 
or total-internal-reflection microscopy, although recently applied to drop impact [24,25,27-33], is not appropriate to track the steep and complex profiles of the L-V interface either.

In this study we adopt ultrafast X-ray imaging that allows us to directly visualize the L-V interface profiles during drop impact on a superheated surface. We observe the formation of a large vapor cavity beneath the Leidenfrost drop and subsequently discover the downward ejection of a jet into the cavity during drop receding. We reveal that the cavity is induced mostly by the retraction of the drop and that the jetting is caused by the convergence of capillary waves along the liquid-cavity interface. We derive a jetting criterion based on viscous dissipation of capillary waves: [Oh $\left.\mathrm{We}^{2}\right] \leqslant$ $66 \pm 10$, where Oh is the Ohnesorge number and We is the Weber number. This criterion is in good agreement with experimental data for various liquids.

\section{EXPERIMENT}

To clearly visualize the L-V interface profiles in the dynamic Leidenfrost phenomenon, we utilize ultrafast X-ray imaging coupled with a drop impact setup [10,19,21,33,34]. The experiments were performed at the 32-ID undulator beamline of the Advanced Photon Source, Argonne National Laboratory, to achieve high temporal $(3.7 \mu \mathrm{s} /$ frame $)$ and spatial $(2 \mu \mathrm{m} / \mathrm{pixel})$ resolutions using a bright white $\mathrm{x}$-ray source $\left(10^{14} \mathrm{ph} / \mathrm{s} / \mathrm{mm}^{2} / 0.1 \%\right.$ bw) $[10,19,21,33,34]$. Figure 1 (a) shows the setup of $\mathrm{x}$-ray imaging conducted in this research. The $\mathrm{x}$-ray imaging system consists of a fast scintillator (LuAG:Ce, decay time $\cong 50 \mathrm{~ns}$ ) and a mirror coupled to a high-speed camera (Photron Fastcam SA-Z) via a $10 \mathrm{x}$ objective lens $[\mathrm{NA}($ numerical aperture $)=0.21]$. The optical axis of the $\mathrm{x}$-ray beam is horizontal, parallel to the heated substrate's surface. X-rays are mostly transmitted through the liquid drop. The camera is synchronized to the x-ray pulses delivered by the synchrotron source to enable direct visualization of the L-V interfaces with high spatial and temporal resolutions, with a $472 \mathrm{~ns}$ exposure time for each frame. The hard $\mathrm{x}$-ray irradiation does not significantly affect the properties of liquids for very short exposures $(<300 \mu \mathrm{s})$ [35].

We employed ethanol, methanol, 1 propanol, 2 propanol, 1 butanol, and 1 pentanol liquid drops (radius $R \approx 1 \mathrm{~mm}$ ) with relatively low latent heats $(L=648-1173 \mathrm{~kJ} / \mathrm{kg}$ ), which facilitate sufficiently thick vapor spaces to be easily captured with an x-ray imaging setup. Liquid drops were dispensed from a $27 \mathrm{G}$ syringe needle (outer diameter: $0.46 \mathrm{~mm}$, inner diameter: $0.25 \mathrm{~mm}$ ) at relatively low heights $(H)$ from 3.3 to $10 \mathrm{~cm}$. The height range corresponds to Weber number $\mathrm{We}=2 \rho R U_{0}^{2} / \sigma=42-140$, where $\rho$ is the liquid density, $U_{0}$ is the impact velocity, and $\sigma$ is the surface tension of the liquid. This range of Weber number is appropriate to demonstrate the propagation of capillary waves in relation to the dynamic Leidenfrost phenomenon, as the dynamic Leidenfrost temperatures are reachable with our setup and the drops are not fragmented after impact. A laser triggering system was installed to sense the falling drop and to trigger the camera and the fast shutter to take the images [10,19,21,33,34]. Silicon wafers of $1 \mathrm{~mm}$ thickness were used as a substrate and heated by a substrate heater (SU-200-IH, Maivac). The substrate temperature $\left(T_{s}\right)$ was measured by a thermocouple (UNI-T UT325) and controlled within $1 \%$ temperature variation from $400{ }^{\circ} \mathrm{C}$ to $600{ }^{\circ} \mathrm{C}$, corresponding with the film boiling regimes for all liquids tested. Cooling of the substrate during the timescale of drop impact is negligible: the thermal timescale ( $\tau_{\mathrm{th}}=k_{s} \rho_{s} C_{p} h^{-2} \cong 10^{-2} \mathrm{~s}$, where $k_{s}$ is the thermal conductivity, $\rho_{s}$ is the substrate density, $C_{p}$ is the specific heat at constant pressure, and $h$ is the heat transfer coefficient from the solid to the drop) is much larger than the impact timescale $\left(2 R / U_{0} \cong 10^{-4} \mathrm{~s}\right)$ [30]. The physical properties of the liquids are shown in Table I [36-39].

\section{RESULTS AND DISCUSSION}

\section{A. Cavity formation and jetting}

Figure 1(b), taken with a field of view (FOV) of $1.5 \mathrm{~mm}$, shows the dynamics of the L-V interface for a Leidenfrost drop, exemplarily shown for an ethanol drop $\left(H=6 \mathrm{~cm}\right.$ and $\left.T_{s}=400{ }^{\circ} \mathrm{C}\right)$. During the transient dynamics of the Leidenfrost drop (see Fig. 1 of the Supplemental Material [40]), as 


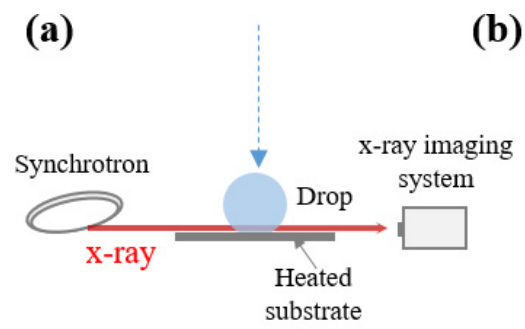

(c)
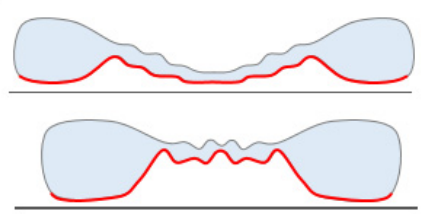

vapor cavity
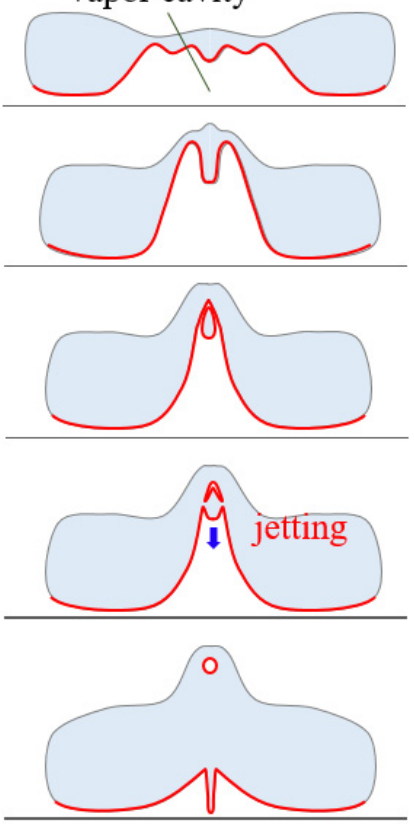

(b)
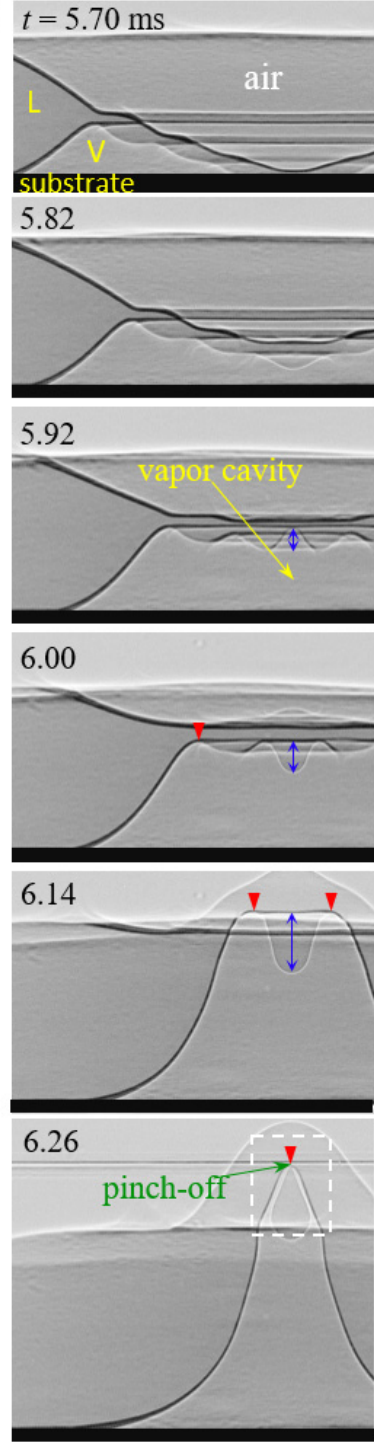
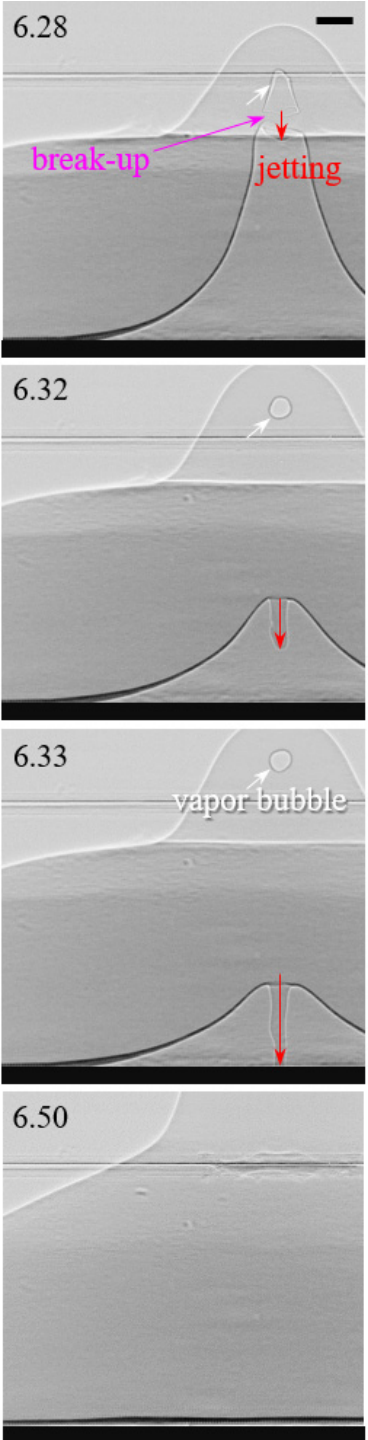

FIG. 1. Ultrafast imaging for jetting by capillary waves in a Leidenfrost drop. (a) Schematic of ultrafast $\mathrm{x}$-ray imaging coupled with the experimental setup of drop impact. (b) Sequential x-ray images, taken with a field of view of $1.5 \mathrm{~mm}$, in ethanol drop impact from $6 \mathrm{~cm}$ height on a heated substrate $\left(T_{s}=400^{\circ} \mathrm{C}\right)$, showing the jetting by capillary waves. L and V mean liquid drop and vapor region, respectively. (c) Illustration of the cavity formation and jetting by the convergence of capillary waves. Here the time at the impact moment is set to $t=0$. Scale bar $100 \mu \mathrm{m}$.

previously reported, a vapor layer develops beneath the liquid drop and capillary waves generate and propagate along the $\mathrm{L}-\mathrm{V}$ interface as the vapor layer thickness reaches a certain value (brown arrows from $t=0.99 \mathrm{~ms}$ in Supplemental Fig. 1) [21,40]. Notably, from the toroidal vapor regions (V) $[t=5.70 \mathrm{~ms}$ in Fig. 1(b)] generated by capillary waves [21], a large vapor cavity develops beneath the center of the drop [yellow arrow at $t=5.92 \mathrm{~ms}$ in Fig. 1(b)], mostly due to the retraction of the liquid rim (L). Here the vapor cannot easily escape outward underneath the heavy rim. The overall height of the cavity gradually increases with retraction (from $t=5.92$ to $6.14 \mathrm{~ms}$ in Fig. 1(b) and Movie 1 in the Supplemental Material [40]). 
TABLE I. Physical properties of methanol, ethanol, 1 propanol, 2 propanol, 1 butanol, and 1 pentanol at room temperature and atmospheric pressure [35-38].

\begin{tabular}{lcccccc}
\hline \hline & Methanol & Ethanol & 1 Propanol & 2 Propanol & 1 Butanol & 1 Pentanol \\
\hline $\begin{array}{l}\text { Density, } \\
\rho\left(\mathrm{kg} / \mathrm{m}^{3}\right)\end{array}$ & 792 & 790 & 803 & 785 & 810 & 818 \\
$\begin{array}{l}\text { Surface tension, } \\
\sigma(\mathrm{N} / \mathrm{m})\end{array}$ & $22.07 \times 10^{-3}$ & $21.97 \times 10^{-3}$ & $23.75 \times 10^{-3}$ & $22.11 \times 10^{-3}$ & $26.28 \times 10^{-3}$ & $25.5 \times 10^{-3}$ \\
$\begin{array}{l}\text { Viscosity, } \\
\mu(\text { Pa s })\end{array}$ & $0.54 \times 10^{-3}$ & $1.07 \times 10^{-3}$ & $2.26 \times 10^{-3}$ & $2.04 \times 10^{-3}$ & $3.61 \times 10^{-3}$ & $4.09 \times 10^{-3}$ \\
\hline \hline
\end{tabular}

The propagating capillary waves on the liquid-cavity interface start to converge at the top center of the cavity (roughly above the impact point or symmetry axis of the drop, $t=5.92 \mathrm{~ms}$ ). The oscillation amplitudes of the converged waves gradually increase (blue arrows at $t=5.92,6.00$, and $6.14 \mathrm{~ms}$ ). Next, by the convergence of the outermost crests (red arrow heads from $t=6.00$ to $6.26 \mathrm{~ms}$ ), we find a pinch-off of the liquid that has been confined within the troughs of the waves (see green arrow at $t=6.26 \mathrm{~ms}$ ). Here the thickness of the cavity wall in the top region (white dashed

(a)
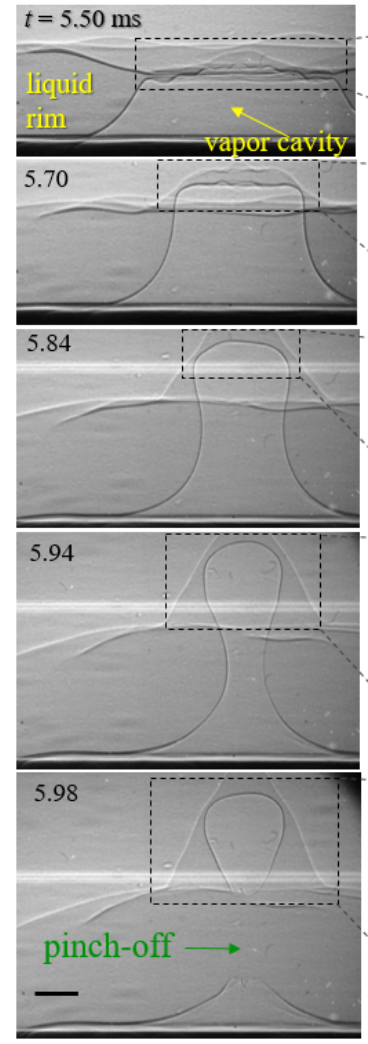

(b)
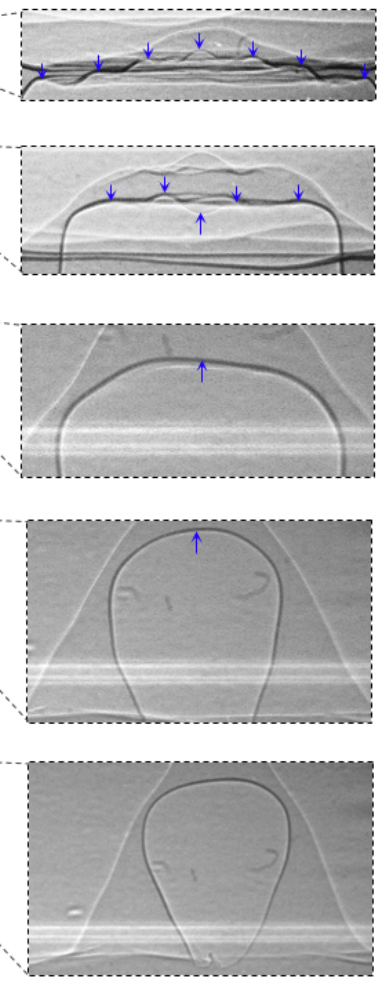
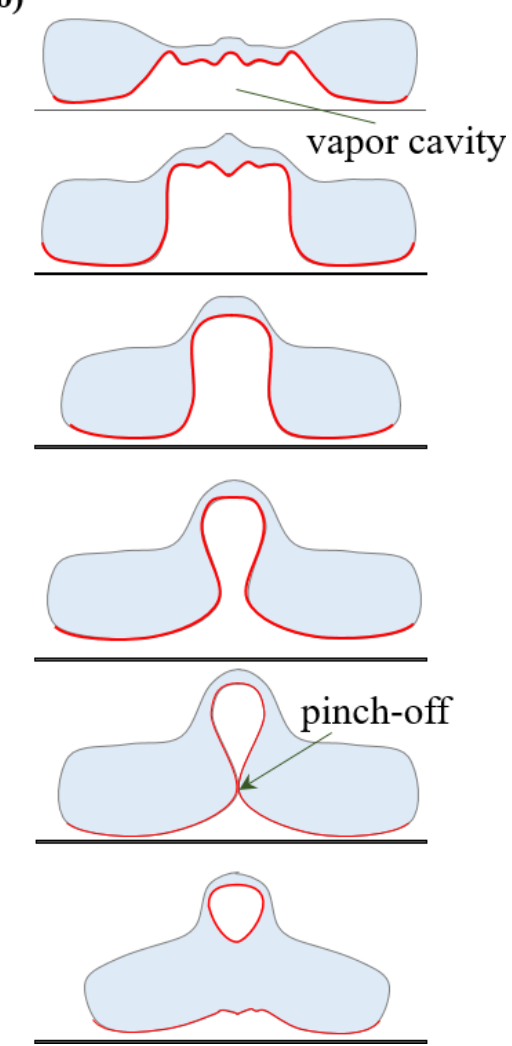

FIG. 2. Ultrafast imaging for no jetting in a Leidenfrost drop. (a) Sequential x-ray images, taken in ethanol drop impact from $8 \mathrm{~cm}$ height on a heated substrate $\left(T_{s}=400^{\circ} \mathrm{C}\right)$. There is no jetting by viscous dissipation of capillary waves while the vapor cavity is pinched-off by the retraction of the liquid rim. (b) Illustration of the no jetting and the pinch-off. Scale bar $100 \mu \mathrm{m}$. 


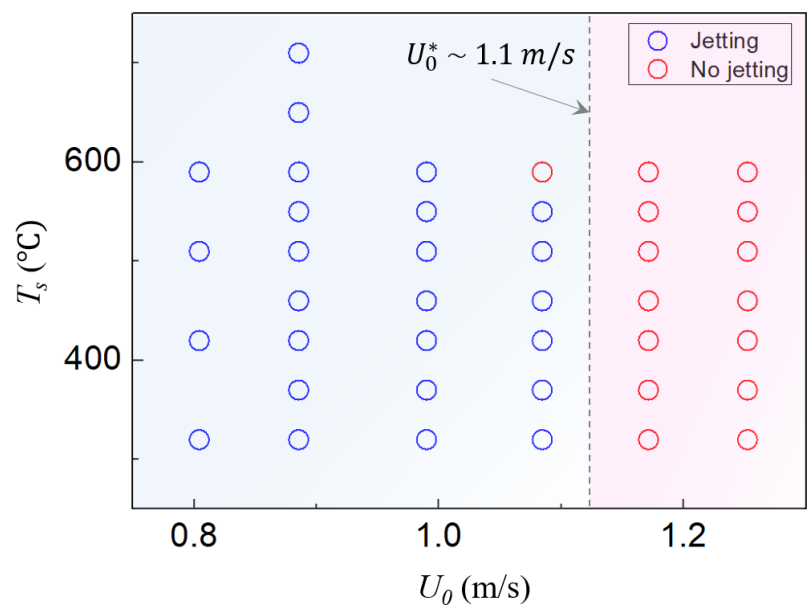

FIG. 3. Phase diagram for jetting and no jetting in terms of $T_{s}$ and $U_{0}$. Jetting and no jetting are mostly differentiated by a boundary of $U_{0} \cong 1.1 \mathrm{~m} / \mathrm{s}$ regardless of $T_{s}$, as tested from $320^{\circ} \mathrm{C}-710^{\circ} \mathrm{C}$. Five to ten experimental repetitions were performed for each data point to verify the repeatability.

box at $t=6.26 \mathrm{~ms}$ ) is too thin to maintain its shape. Then the top region of the cavity may break up (magenta arrow at $t=6.28 \mathrm{~ms}$ ) by Rayleigh instability [41], forming a trapped vapor bubble (white arrows at $t=6.28-6.33 \mathrm{~ms}$ ). At the moment of the breakup, the liquid droplet recoalesces with the pinched-off liquid (at $t=6.28 \mathrm{~ms}$ ). The convergence of capillary waves, followed by the coalescence, induces the momentum transfer, finally leading to the downward ejection of a jet into the cavity (red arrows from $t=6.28$ to $6.33 \mathrm{~ms}$ ), followed by the collapse of the cavity $(t=6.50 \mathrm{~ms}$ ). Figure 1(c) schematically depicts the overall dynamics of the cavity, from the toroidal vapor region to the cavity formation and to the downward jetting by the convergence of capillary waves, which is consistently observed at low impact heights $(H \leqslant 6 \mathrm{~cm})$.

\section{B. No jetting}

Interestingly, the jetting by capillary waves is not observed for high impact heights $(H>6 \mathrm{~cm})$, as demonstrated in Fig. 2(a) and Supplemental Movie 2 [40]. A vapor cavity is still formed beneath the center of the drop [yellow arrow at $t=5.50 \mathrm{~ms}$ in Fig. 2(a)], increasing in its height with time, again due to the retraction of the liquid rim. However, capillary waves on the liquid-cavity interface become gradually damped out, as demonstrated by blue arrows from $t=5.50$ to $5.84 \mathrm{~ms}$ in Fig. 2(a). Thus, jetting by the convergence of capillary waves does not occur for high impact heights. Apart from that, the retraction continues until a large top part of the vapor cavity is pinched-off not far above the bottom of the cavity due to recoalescence of the inward-propagating rims (green arrow, $t=5.98 \mathrm{~ms}$ ). This will lead to a different type of jet, described in Sec. III D. The overall dynamics in the absence of the jetting by capillary waves is depicted schematically in Fig. 2(b).

We investigate the jetting by capillary waves for various impact velocities $\left(U_{0}\right)$ and substrate temperature $\left(T_{s}\right)$, as demonstrated for ethanol drops in Fig. 3. We note that there is a boundary $\left(U_{0}: 1.1 \mathrm{~m} / \mathrm{s}\right)$ in $U_{0}$ between jetting and no jetting, regardless of $T_{s}$. Each point is deduced by repeated five to ten experiments.

Let us now address the absence of jetting due to capillary waves a bit more quantitatively. No jetting in high impact velocities can be explained by viscous dissipation of capillary waves. Usually, jetting by capillary waves occurs due to the convergence of capillary waves along the liquid/air interface $[9,10,16-18]$. However, jetting can be inhibited if the capillary waves are significantly attenuated by viscous dissipation $[9,10]$. In general, the attenuation of the wave amplitude $A(x)$ can 
be written as

$$
A(x)=A_{0} e^{-\alpha x},
$$

where $x$ is the traveling distance of the waves, $A_{0}$ is the initial amplitude at $x=0$, and $\alpha$ is the attenuation coefficient. For viscous damping of capillary waves, $\alpha$ is given by $[42,43]$

$$
\alpha=\frac{8 \pi^{2} \mu}{\rho U_{g} \lambda^{2}},
$$

where $\mu$ is the dynamic viscosity of the liquid, and $\lambda$ and $U_{g}$ are the wavelength and group velocity of the waves, respectively. For other experiments with capillary-wave-induced jetting in the drop impact, $U_{g}$ is known as $[21,44]$

$$
U_{g}=\frac{3}{2} \sqrt{\frac{2 \pi \sigma}{\rho \lambda}} .
$$

For drop impact on a superheated substrate above the Leidenfrost point, Eq. (3) can be applied to capillary waves propagated along the vapor-liquid interface [21]. From Eqs. (2) and (3), we obtain the relation between $\alpha$ and $\lambda$ as

$$
\alpha=\sqrt{\frac{128 \pi^{3} \mu^{2}}{9 \rho \sigma \lambda^{3}}} .
$$

Again from Ref. [21] that derives the wavelength of capillary waves in drop impact, we use the scaling

$$
\lambda \propto \frac{\sigma}{\rho U_{0}^{2}} .
$$

Finally, from Eqs. (4) and (5), we can deduce the relation between $\alpha$ and $U_{0}$ as

$$
\alpha \propto U_{0}^{3} \text {. }
$$

This scaling relation shows that the attenuation drastically rises as a cubic function of $U_{0}$, suggesting significant damping of capillary waves at high impact velocities. This explains why no jetting is observed at high impact velocities $\left(U_{0} \geqslant 1.1 \mathrm{~m} / \mathrm{s}\right)$ in Fig. 3 . In fact, we measure the attenuation coefficients of the capillary waves from high-resolution x-ray snapshots for various $U_{0}$ and $T_{s}$ for ethanol drops in Fig. 3 to confirm the relation between $\alpha$ and $U_{0}$, Eq. (6). Specifically, we measure the amplitudes, $A_{1}$ and $A_{2}$, of the first and the second peaks, respectively, and the wavelength $\lambda$ [see the inset of Fig. 4(a)]. Here $A_{2}$ is smaller than $A_{1}$, attributed to the damping of the capillary waves. Then $\alpha$ can be written using Eq. (1) as follows:

$$
\alpha=\ln \left(A_{1} / A_{2}\right) / \lambda \text {. }
$$

As seen in Fig. 4(a), the measured attenuation coefficient (blue squares) using Eq. (7) very well agrees with the calculated one (red circles) using Eq. (4) for all impact velocities tested. Here the attenuation coefficients calculated (red circles) are obtained by measuring the $\lambda$ values from $\mathrm{x}$-ray snapshots and inserting the measured values into Eq. (4).

We used the material properties at room temperature from Table I. Indeed, the measured attenuation coefficient drastically increases with $U_{0}$. The dashed line is the best fit with an allometric scaling $\left(\alpha \propto U_{0}^{p}\right.$ ) where the exponent $p$ is $2.88 \pm 0.21$, well matched with Eq. (6). One would expect that the measured attenuation coefficients should depend on the substrate temperature $T_{s}$. However, we find that they are practically temperature independent, as seen in the plot of $\alpha$ vs $T_{s}$ in Fig. 4(b), and by comparing the measured values (blue squares) to those (red circles) calculated based on room temperature properties of the liquid. On the one hand, this supports the assumption that the liquid at the bottom surface of a Leidenfrost drop retains a roughly constant temperature, no matter what the substrate temperature is. The attenuation coefficient that depends on the liquid 
(a)

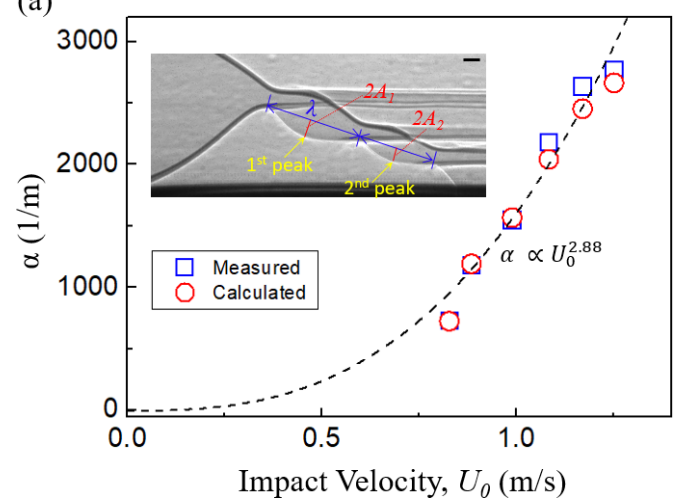

(b)

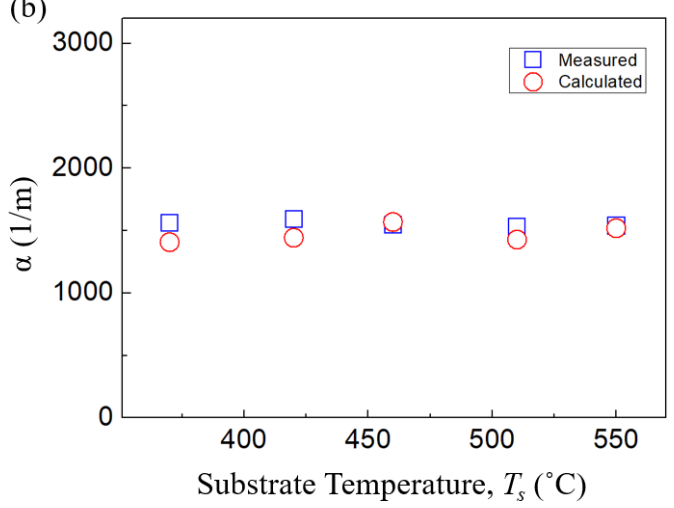

FIG. 4. Attenuation coefficient of capillary waves. (a) Comparison of the measured (blue squares) and calculated (red circles; based on viscous damping) attenuation coefficients $(\alpha)$ at various impact velocities $\left(U_{0}\right)$ and $T_{s}=460^{\circ} \mathrm{C}$ for ethanol drops. The dashed line is the allometric scaling for the measured values, $\alpha \propto U_{0}^{p}$, where the exponent $p$ is estimated as $2.88 \pm 0.21$. The inset shows how to measure the amplitudes of the first and second peaks from x-ray images. Scale bar in the inset $100 \mu \mathrm{m}$. (b) The measured (blue squares) and calculated (red circles) attenuation coefficients at different substrate temperatures $\left(T_{s}\right)$ for $U_{0}=1 \mathrm{~m} / \mathrm{s} . \alpha$ is invariant with $T_{s}$.

properties including the liquid temperature is thus independent of the substrate temperature or conduction through the vapor or the liquid drop. On the other hand, it is astonishing considering the large sizes of the cavities and the inhomogeneous temperature distribution within spreading and retracting drops. The results of Figs. 4(a) and 4(b) confirm that the absence of jetting at high impact velocities is mostly due to viscous damping of the capillary waves.

\section{Critical condition for jetting}

Now we develop a general condition for the occurrence of jetting by capillary wave convergence. In general, jetting occurs through the convergence of capillary waves, followed by a significant momentum transfer downward, as expressed by $D t<1$, where $D$ is the damping rate of the waves $[9,10]$. This inequality equation can be written as $\alpha x<1$, since $D=\alpha U_{g}$ and $U_{g}=x / t$. Here, the traveling distance of the waves $x$ is approximated to the maximum radius of the liquid drop $R \mathrm{We} \mathrm{e}^{0.5}$, where We is the Weber number $\left(=2 \rho R U_{0}^{2} / \sigma\right)$. From Eqs. (4) and (5), $\alpha x$ can be estimated with nondimensional numbers, the Ohnesorge number $(\mathrm{Oh}=\mu / \sqrt{2 \rho \sigma R})$ and the Weber number:

$$
\alpha x=c \sqrt{\frac{\mu^{2}}{2 \rho \sigma \mathrm{R}}} \sqrt{\left[\frac{2 \rho R U_{0}^{2}}{\sigma}\right]^{4}}=c \mathrm{OhWe}^{2},
$$

where $c$ is a constant. Then the relationship, $\mathrm{OhWe}^{2}<1 / c$ (i.e., $\alpha x<1$ ), suggests the existence of a critical number, $\left[\mathrm{OhWe}^{2}\right]^{*}$, above which jetting caused by capillary waves is absent due to viscous damping by dissipating capillary waves. In the case of ethanol drops, $\left[\mathrm{OhWe}^{2}\right]^{*}$ is measured as 56 using $U_{0}^{*}(=1.1 \mathrm{~m} / \mathrm{s})$ in Fig. 3 and the physical properties of ethanol in Table I.

To generalize the critical number, we test various liquids (methanol, ethanol, 1 propanol, 2 propanol, 1 butanol, and 1 pentanol) with different viscosities $\left(\mu=0.54-4.09 \times 10^{-3} \mathrm{~Pa} \mathrm{~s}\right)$ or Oh numbers $(\mathrm{Oh}=0.0109-0.0289)$ and various impact velocities $\left(U_{0}=0.8-1.4 \mathrm{~m} / \mathrm{s}\right)$ or We numbers (We $=42-140)$. All the droplets have similar radii, $R \approx 1 \mathrm{~mm}$. A phase diagram for the occurrence of jetting and no jetting is obtained in terms of $U_{0}$ and $\mathrm{OhWe}^{2}$, as demonstrated in Fig. 5(a). Each point is deduced by repeating five to ten experiments. From this extended data set we observe that the critical number for jetting is invariant, $\left[\mathrm{OhWe}^{2}\right]^{*} \approx 66 \pm 10$, regardless of liquids employed or 

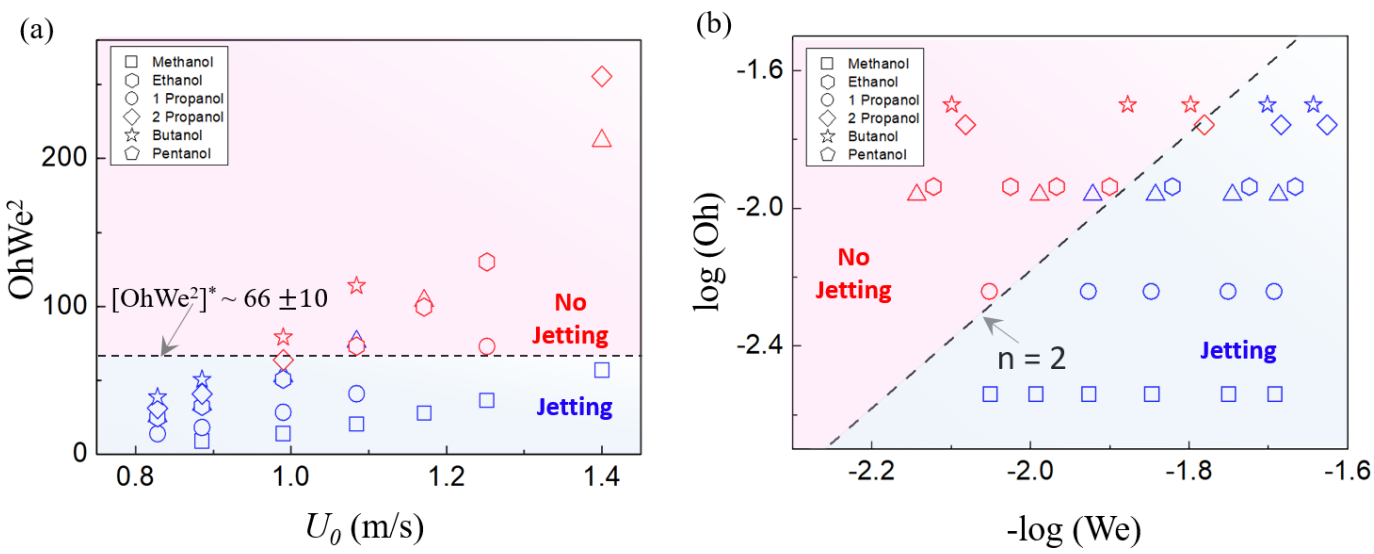

FIG. 5. Condition for jetting and no jetting. (a) Phase diagram for jetting and no jetting in terms of $\mathrm{OhWe}^{2}$ and $U_{0}$. It shows a general critical condition for the jetting by capillary waves, regardless of liquids, as tested for methanol, ethanol, 1 propanol, 2 propanol, 1 butanol, and 1 pentanol. Dashed line represents the critical jetting condition: $\left[\mathrm{OhWe}^{2}\right] \leqslant 66 \pm 10$. Five to ten experimental repetitions were performed for each data point to verify the repeatability. (b) $\log (\mathrm{Oh})$ vs $-\log (\mathrm{We})$ plot (with subscript 10$)$ to validate the exponent $(n)$ of Weber number in Eq. (9).

impact velocities. For drop impacts at $\mathrm{OhWe}^{2}<66 \pm 10$, as seen in Fig. 5(a), we always observe the downward jetting by capillary waves. By taking the condition $\left[\mathrm{OhWe}^{2}\right]^{*} \approx 66 \pm 10$, we get

$$
\log (\mathrm{Oh})=-2 \log (\mathrm{We})+C
$$

where $C=\log (66) \sim 1.82$ in these experiments (using log with subscript 10). Equation (9) suggests the proper exponent of We for the critical jetting condition to be $n=2$, which well matches the $\log (\mathrm{Oh})$ vs $-\log (\mathrm{We})$ plot in Fig. 5(b).

\section{Jetting by the liquid rims}

In this section we discuss a different type of jetting caused by the recoalescence of the liquid rims. An impacting liquid drop initially spreads due to the inertia but later retracts toward the center due to the surface tension (Supplemental Fig. 1). As measured from ultrafast X-ray snapshots during retraction, we observe that the group velocity of capillary waves is higher than the retraction velocity by $U_{g}(0.75-0.85 \mathrm{~m} / \mathrm{s})>v_{\text {retraction }}(0.52-0.66 \mathrm{~m} / \mathrm{s})$, regardless of impact heights. It is then conceivable that the convergence of capillary waves occurs before the convergence of the liquid rims, as seen in Fig. 1(b) $(t=5.92-6.26 \mathrm{~ms})$, which explains the downward jetting in the cavity and thus the collapse of the cavity [Fig. 1(b)]. However, if the capillary waves are significantly damped out, as in Fig. 2(a), the liquid rims can recoalesce at the center, thus pinching off a large top part of the cavity, without jetting by capillary waves [Fig. 2(a)]. Then, the coalescence of the liquid rims causes a different type of jet, specifically, both upward and downward jetting into the pinched-off regions, as indicated by the red arrows (at $t=6.59,6.61$, and $6.64 \mathrm{~ms}$ ) in Fig. 6 . The velocities of the jets by the liquid rims, measured as 10-20 m/s, are much higher than those of the jets by capillary waves [as demonstrated in Fig. 1(b)], measured as $2-3 \mathrm{~m} / \mathrm{s}$, mostly due to much higher energy and momentum transfer by the collision convergence of the liquid rims compared to that by capillary waves. This type of jet is similar to the high-velocity jets observed in the pinched-off air cavity during the impact of a liquid disk on a liquid pool [45]. Further analytical study regarding velocities of jets depending on types of jetting is necessary. 

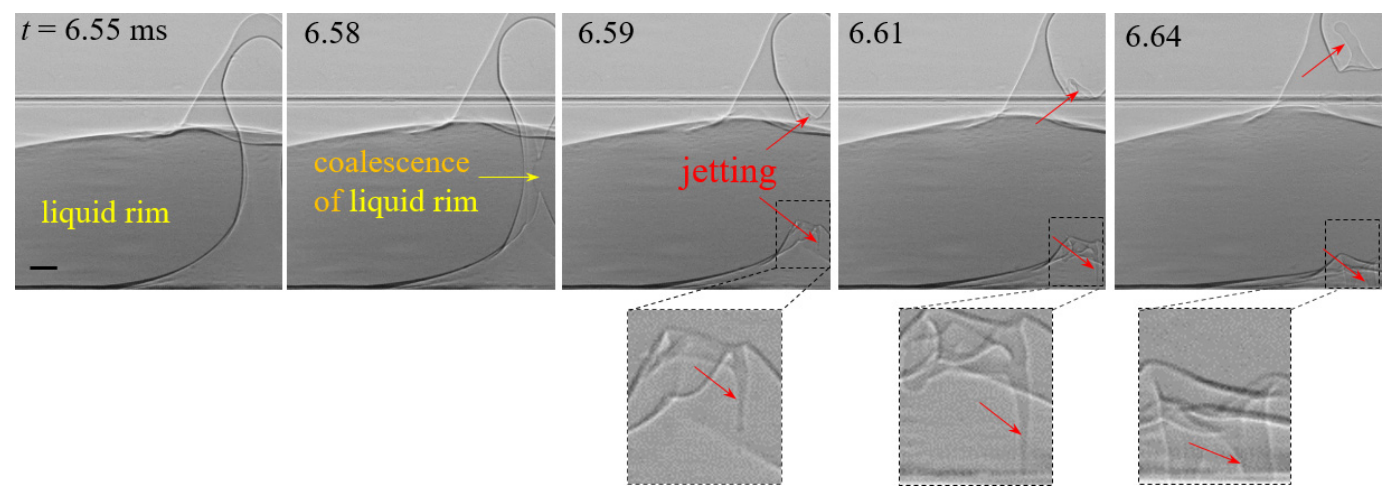

FIG. 6. Representative x-ray images for different types of jetting by the liquid rims, taken at ethanol drop impact from $8 \mathrm{~cm}$ height on a heated substrate $\left(T_{s}=400^{\circ} \mathrm{C}\right)$. Both upward and downward jetting occur by the energy transfer at the moment of the convergence of the liquid rims. Scale bar $100 \mu \mathrm{m}$.

\section{CONCLUSION}

In conclusion, we find downward jetting of a Leidenfrost drop caused by capillary waves propagating on the bottom liquid-vapor interface of the retracting drop. Using ultrafast $\mathrm{x}$-ray imaging, we have observed the formation of a large vapor cavity beneath the Leidenfrost drop and then downward ejection of a jet into the cavity. We reveal that the cavity is induced mostly by the retraction of the drop. One main mechanism of the jetting is the convergence of capillary waves propagating along the liquid-cavity interface. This jetting can be inhibited by viscous damping of the capillary waves, which is clearly observed at high impact heights. We establish a phase diagram for jetting in terms of the impact velocity $U_{0}$ and dimensionless group [OhWe ${ }^{2}$, and suggest a jetting criterion based on viscous dissipation of capillary waves: $\left[\mathrm{OhWe}^{2}\right] \leqslant 66 \pm 10$. These results can offer substantial insight for further analytical, numerical, and experimental works on jetting or capillary waves, which can be helpful for understanding many different industrial applications such as spray cooling, fuel combustion, and inkjet printing.

\section{ACKNOWLEDGMENTS}

This research was supported by the National Research Foundation of Korea (NRF) through a grant funded by the Korean Government (Grant No. NRF-2017R1E1A1A01075274), the Brain Korea 21 PLUS Project for the Center for Creative Industrial Materials, and the German Science Foundation (Deutsche Forschungsgemeinschaft, Contracts No. HA 8467/1 and No. 8467/3). This work is also part of an Industrial Partnership Programme of the Foundation for Fundamental Research on Matter (FOM), which is financially supported by the Netherlands Organisation for Scientific Research (NWO). This research program is cofinanced by Oceé Technologies B.V., University of Twente, and Eindhoven University of Technology. The use of the Advanced Photon Source, an Office of Science User Facility operated for the U.S. Department of Energy (DOE) by the Argonne National Laboratory, was supported by the U.S. DOE under Contract No. DE-AC02$06 \mathrm{CH} 11357$.

[1] Y. S. Joung and C. R. Buie, Aerosol generation by raindrop impact on soil, Nat. Commun. 6, 6083 (2015).

[2] A. van der Bos, M.-J. van der Meulen, T. Driessen, M. van den Berg, H. Reinten, H. Wijshoff, M. Versluis, and D. Lohse, Velocity Profile Inside Piezoacoustic Inkjet Droplets in Flight: Comparison Between Experiment and Numerical Simulation, Phys Rev. Appl. 1, 014004 (2014). 
[3] S.-J. Chen and A. A. Tseng, Spray and jet cooling in steel rolling, Int. J. Heat Fluid Flow 13, 358 (1992).

[4] A. Moreira, A. Moita, and M. Panao, Advances and challenges in explaining fuel spray impingement: How much of single droplet impact research is useful? Prog Energy Combust. Sci. 36, 554 (2010).

[5] G. G. Wells, R. Ledesma-Aguilar, G. McHale, and K. Sefiane, A sublimation heat engine, Nat. Commun. 6, 6390 (2015).

[6] J. C. Bird, R. De Ruiter, L. Courbin, and H. A. Stone, Daughter bubble cascades produced by folding of ruptured thin films, Nature 465, 759 (2010).

[7] G. B. Deane and M. D. Stokes, Scale dependence of bubble creation mechanisms in breaking waves, Nature (London) 418, 839 (2002).

[8] A. M. Worthington, A Study of Splashes (Longmans, Green, and Company, London, 1908).

[9] F. Blanchette and T. P. Bigioni, Partial coalescence of drops at liquid interfaces, Nat. Phys. 2, 254 (2006).

[10] J. S. Lee, B. M. Weon, S. J. Park, J. H. Je, K. Fezzaa, and W.-K. Lee, Size limits the formation of liquid jets during bubble bursting, Nat. Commun. 2, 367 (2011).

[11] L. Rayleigh, On the capillary phenomena of jets, Proc. R. Soc. London 29, 71 (1879).

[12] G.-J. Michon, C. Josserand, and T. Séon, Jet dynamics post drop impact on a deep pool, Phys. Rev. Fluids 2, 023601 (2017).

[13] A. L. Yarin, Drop impact dynamics: Splashing, spreading, receding, bouncing, Annu. Rev. Fluid Mech. 38, 159 (2006).

[14] E. Ghabache and T. Séon, Size of the top jet drop produced by bubble bursting, Phys. Rev. Fluids 1, 051901 (2016).

[15] J. M. Gordillo and S. Gekle, Generation and breakup of Worthington jets after cavity collapse, Part 2. Tip breakup of stretched jets, J. Fluid Mech. 663, 331 (2010).

[16] L. J. Leng, Splash formation by spherical drops, J. Fluid Mech. 427, 73 (2001).

[17] B. Ray, G. Biswas, and A. Sharma, Regimes during liquid drop impact on a liquid pool, J. Fluid Mech. 768, 492 (2015).

[18] M. K. Tan, J. R. Friend, and L. Y. Yeo, Interfacial Jetting Phenomena Induced by Focused Surface Vibrations, Phys. Rev. Lett. 103, 024501 (2009).

[19] J. S. Lee, B. M. Weon, J. H. Je, and K. Fezzaa, How Does an Air Film Evolve into a Bubble During Drop Impact? Phys. Rev. Lett. 109, 204501 (2012).

[20] S. Thoroddsen, T. Etoh, K. Takehara, N. Ootsuka, and Y. Hatsuki, The air bubble entrapped under a drop impacting on a solid surface, J. Fluid Mech. 545, 203 (2005).

[21] S.-H. Lee, S. J. Lee, J. S. Lee, K. Fezzaa, and J. H. Je, Transient dynamics in drop impact on a superheated surface, Phys. Rev. Fluids 3, 124308 (2018).

[22] J. G. Leidenfrost, De Aquae Communis Nonnullis Qualitatibus Tractatus (Johann Straube, Duisburg, 1756).

[23] D. Quéré, Leidenfrost dynamics, Annu. Rev. Fluid Mech. 45, 197 (2013).

[24] M. Shirota, M. A. van Limbeek, C. Sun, A. Prosperetti, and D. Lohse, Dynamic Leidenfrost Effect: Relevant Time and Length Scales, Phys. Rev. Lett. 116, 064501 (2016).

[25] T. Tran, H. J. Staat, A. Prosperetti, C. Sun, and D. Lohse, Drop Impact on Superheated Surfaces, Phys. Rev. Lett. 108, 036101 (2012).

[26] M. M. Driscoll, C. S. Stevens, and S. R. Nagel, Thin film formation during splashing of viscous liquids, Phys. Rev. E 82, 036302 (2010).

[27] M. M. Driscoll and S. R. Nagel, Ultrafast Interference Imaging of Air in Splashing Dynamics, Phys. Rev. Lett. 107, 154502 (2011).

[28] J. M. Kolinski, L. Mahadevan, and S. M. Rubinstein, Lift-Off Instability During the Impact of a Drop on a Solid Surface, Phys. Rev. Lett. 112, 134501 (2014).

[29] M. Khavari and T. Tran, Universality of oscillating boiling in Leidenfrost transition, Phys. Rev. E 96, 043102 (2017).

[30] M. A. van Limbeek, M. Shirota, P. Sleutel, C. Sun, A. Prosperetti, and D. Lohse, Origin of spray formation during impact on heated surfaces, Int. J. Heat Mass Transfer 97, 101 (2016).

[31] S.-H. Lee, K. Harth, M. Rump, M. Kim, D. Lohse, K. Fezzaa, and J. H. Je, Drop Impact on Hot Plates: Contact Times, Lift-Off and the Lamella Rupture, arXiv:2003.07412. 
[32] H. Nair, H. J. Staat, T. Tran, A. van Houselt, A. Prosperetti, D. Lohse, and C. Sun, The Leidenfrost temperature increase for impacting droplets on carbon-nanofiber surfaces, Soft Matter 10, 2102 (2014).

[33] J. S. Lee, S. J. Park, J. H. Lee, B. M. Weon, K. Fezzaa, and J. H. Je, Origin and dynamics of vortex rings in drop splashing, Nat. Commun. 6, 8187 (2015).

[34] G. C. Lee, H. Noh, H. J. Kwak, T. K. Kim, H. S. Park, K. Fezzaa, and M. H. Kim, Measurement of the vapor layer under a dynamic Leidenfrost drop, Int. J. Heat Mass Transfer 124, 1163 (2018).

[35] B. Weon, J. H. Je, Y. Hwu, and G. Margaritondo, Decreased Surface Tension of Water by Hard-X-Ray Irradiation, Phys. Rev. Lett. 100, 217403 (2008).

[36] U.S. Coast Guard, Chemical Hazard Response Information System (CHRIS) - Hazardous Chemical Data, Commandant Instruction (U.S. Government Printing Office, Washington, DC, 1999), p. 16465.12C.

[37] W. M. Haynes, CRC Handbook of Chemistry and Physics, 95th ed. (CRC Press, Boca Raton, FL, 2014-2015), pp. 6-231.

[38] Kirk-Othmer, Encyclopedia of Chemical Technology (John Wiley and Sons, New York, 1991-Present), Vol. 1.

[39] ILO International Chemical Safety Cards (ICSC), http://www.ilo.org/dyn/icsc/showcard.display?p_ version $=2 \&$ p_card_id $=0535$.

[40] See Supplemental Material at http://link.aps.org/supplemental/10.1103/PhysRevFluids.5.074802 for supplemental figure for understanding the transient dynamics and movies for understanding jetting dynamics.

[41] J. W. Strutt and L. Rayleigh, On the instability of jets, Proc. London Math. Soc. 10, 4 (1878).

[42] F. Behroozi, B. Lambert, and B. Buhrow, Direct measurement of the attenuation of capillary waves by laser interferometry: Noncontact determination of viscosity, Appl. Phys. Lett. 78, 2399 (2001).

[43] F. Behroozi, J. Smith, and W. Even, Stokes' dream: Measurement of fluid viscosity from the attenuation of capillary waves, Am. J. Phys. 78, 1165 (2010).

[44] Y. Renardy, S. Popinet, L. Duchemin, M. Renardy, S. Zaleski, C. Josserand, M. A. Drumright-Clarke, D. Richard, C. Clanet, and D. Quéré, Pyramidal and toroidal water drops after impact on a solid surface, J. Fluid Mech. 484, 69 (2003).

[45] S. Gekle and J. M. Gordillo, Generation and breakup of Worthington jets after cavity collapse, Part 1. Jet formation, J. Fluid Mech. 663, 293 (2010). 